\title{
The Emerging Importance of Evaluation of Pre-Injury Sleep Characteristics among Adolescents with Traumatic Brain Injury
}

\section{Erik Everhart*, James P Loveless and Alexandra J Stephenson \\ Department of Psychology, East Carolina University, Greenville, NC 27858, USA}

Sports-related concussions (SRC) are common, with 1.6 to 3.8 million occurring within the United States each year [1]. Second to vehicular trauma, SRC is a leading cause of traumatic brain injury among individuals within the ages of 15-24 [2]. While most adolescents recover from concussion, a subset displays or experiences an increase in reported post concussive symptoms. It is thought that these symptoms are in part related to pre-injury neurological and psychosocial vulnerability, as well as post-injury decrements in neuropsychological and neurobehavioral functioning [3]. The pre-injury psychosocial vulnerabilities are numerous and include psychological disturbance, personality traits, and coping abilities that ultimately influence rate of improvement, report of residual symptoms, and possibly long-term quality of life [4].

Of the existing pre-injury vulnerabilities, sleep function has received considerably less attention. However, adolescent sleep often lacks duration, quality and consistency [5]. For example, though there is a general consensus among sleep researchers that adolescents require approximately 9 to $10 \mathrm{~h}$ of sleep per night for optimal functioning, only $20 \%$ get the recommended nine hours of sleep on school nights and $45 \%$ get less than eight hours on school nights [6]. The consequences of poor sleep affect a number of domains including school performance, mood regulation, cognitive processes including reaction speed and attention, and overall health [7]. With regard to cognitive processes, it is possible that deficits in psychomotor vigilance are the most pronounced. For instance, deficits in psychomotor vigilance are observed following one night of sleep restriction after 4 and $5 \mathrm{~h}$ of sleep [8].

Given that poor sleep patterns among adolescents are common and that poor sleep quality disrupts cognitive function, it is intuitive that pre-injury sleep patterns and/or disruption may alter sequelae associated with brain injury. Thus, an area of increasing research emphasis is the effect of sleep on neurocognitive function and recovery from SRC.

For example, in one recent study, adolescent athletes with preexisting and self-reported sleep problems (i.e., sleeping less and/ or difficulty falling asleep) who were diagnosed with sports-related concussion (SRC) reported more symptoms on the Post-Concussion Symptom Scale (PCSS) [9] at 5-7 days and 10-14 days after injury than did concussed athletes who did not report sleep problems. These athletes also performed worse on verbal memory testing than did athletes who did not report sleep problems [10]. A more recent study that included over 7000 athletes found a "dose-response" effect of sleep duration the night prior to baseline cognitive testing. Sleep restricted adolescents had poorer performance on tests of verbal memory, visual memory, visual motor speed, and reaction time compared to demographically matched controls [11]. These two studies highlight the importance of understanding and evaluating possible pre-existing sleep problems among adolescents who suffer traumatic brain injuries. These studies are also reflective of an emerging literature base that seeks to examine the influence of common pre-injury vulnerabilities on outcome following traumatic brain injury.

One instrument that may be useful for evaluation of sleep characteristics among adolescents with SRC is the short version of the Adolescent Sleep Wake Scale (ASWS). The ASWS is a 10-item scale with three factors including (a) Falling Asleep and Reinitiating Sleep-Revised, (b) Returning to Wakefulness-Revised, and (c) Going to Bed-Revised. Internal consistency for this three factor model using Cronbach's Alpha ranges from 0.64 to 0.89 using a healthy sample and from 0.74 to 0.84 using a pooled sample of adolescents with chronic pain, sickle cell disease, traumatic brain injury and depressive disorders [12]. Examination of Cronbach's Alpha for a more diverse sample indicates that the ASWS has acceptable reliability for African American, Caucasian, and multi-ethnic adolescents, but not for Latino adolescents [13].

Considerations for future research and clinical practice include the parsing of effects of various sleep characteristics on the sequelae of SRC. For example, emerging research examines the combination of sleep restriction and other sleep problems such as "difficulty falling asleep" [11]. Future studies may examine the effects of acute versus chronic sleep deprivation, individual variables associated with insomnia such as number of awakenings, total sleep time, and time required to fall asleep.

\section{References}

1. Rutland-Brown W, Langlois JA, Thomas KE, Xi YL (2006) Incidence of traumatic brain injury in the United States, 2003. J Head Trauma Rehabil 21: 544-548.

2. Sosin DM, Sniezek JE, Thurman DJ (1996) Incidence of mild and moderate brain injury in the United States, 1991. Brain Inj 10: 47-54.

3. Yeates KO, Luria J, Bartkowski H, Rusin J, Martin L, et al. (1999) Postconcussive symptoms in children with mild closed head injuries. J Head Trauma Rehabil 14: $337-350$.

4. Vanderploeg RD, Curtiss G, Luis CA, Salazar AM (2007) Long-term morbidities following self-reported mild traumatic brain injury. J Clin Exp Neuropsychol 29 585-598.

5. Moran AM, Everhart DE (2012) Adolescent sleep: Review of characteristics consequences and intervention. Journal of Sleep Disorders. Treatment and Care 1: 1-8.

6. Carskadon MA, Harvey K, Duke P, Anders TF, Litt IF, et al. (1980) Pubertal changes in daytime sleepiness. Sleep 2: 453-460.

7. Millman RP; Working Group on Sleepiness in Adolescents/Young Adults; AAP Committee on Adolescence (2005) Excessive sleepiness in adolescents and young adults: Causes, consequences, and treatment strategies. Pediatrics 115: 1774-1786.

8. Belenky G, Wesensten NJ, Thorne DR, Thomas ML, Sing HC, et al. (2003) Patterns of performance degradation and restoration during sleep restriction and subsequent recovery: A sleep dose-response study. J Sleep Res 12: 1-12.

9. Chen JK, Johnston KM, Collie A, McCrory P, Ptito A (2007) A validation of the post-concussion symptom scale in the assessment of complex concussion using cognitive testing and functional MRI. Journal of Neurology, Neurosurgery, and Psychiatry 78: 1231-1238.

*Corresponding author: Erik Everhart D, Department of Psychology, East Carolina University Greenville, NC 27858, USA, Tel: 2523284138; E-mail: everhartd@ecu.edu

Received May 19, 2016; Accepted June 03, 2016; Published June 10, 2016

Citation: D Erik Everhart, Loveless JP, Stephenson AJ (2016) The Emerging Importance of Evaluation of Pre-Injury Sleep Characteristics among Adolescents with Traumatic Brain Injury. Int J Neurorehabilitation 3: 210. doi:10.4172/23760281.1000210

Copyright: @ 2016 Erik Everhart D, et al. This is an open-access article distributed under the terms of the Creative Commons Attribution License, which permits unrestricted use, distribution, and reproduction in any medium, provided the original author and source are credited. 
Citation: D Erik Everhart, Loveless JP, Stephenson AJ (2016) The Emerging Importance of Evaluation of Pre-Injury Sleep Characteristics among Adolescents with Traumatic Brain Injury. Int J Neurorehabilitation 3: 210. doi:10.4172/2376-0281.1000210

10. Sufrinko A, Pearce K, Elbin RJ, Covassin T, Johnson E, et al. (2015) The effect of pre-injury sleep difficulties on neurocognitive impairment and symptoms after sport-related concussion. Am J Sports Med 43: 830-838.

11. Sufrinko A, Johnson EW, Henry LC (2016) The influence of sleep duration and sleep-related symptoms on baseline neurocognitive performance among male and female high school athletes. Neuropsychology 30: 484491.
12. Essner B, Noel M, Myrvik M, Palermo T (2015) Examination of the factor structure of the adolescent sleep-wake scale (ASWS). Behav Sleep Med 13: 296-307.

13. Sufrinko AM, Valrie CR, Lanzo L, Bond KE, Trout KL, et al. (2015) Empirical validation of a short version of the adolescent sleep-wake scale using a sample of ethnically diverse adolescents from an economically disadvantage community. Sleep Med 16: 1204-1206. 